\title{
ORIGINAL ARTICLE Neurophysiological assessment of the injured spinal cord: an intraoperative approach
}

\author{
P Costa $^{1,2}$, G Faccani ${ }^{2,3}$, F Sala $^{4}$, E Montalenti $^{2}$, ML Giobbe $^{1,2}$ and V Deletis ${ }^{5}$
}

Study design: Prospective, observational study.

Objectives: To assess the spinal cord function intraoperatively in subjects during spine stabilization for spinal cord trauma, by recording muscular (m-MEPs) and epidural motor evoked potentials (e-MEPs, D wave) along with cortical and epidural somatosensory evoked potentials (e-SEPs) and predicting the outcome of spinal cord injury (SCI).

Setting: Regional Trauma Center, Torino, Italy.

Methods: Fifty-five patients were intraoperatively studied during posterior spine stabilization surgery for traumatic SCl. In all, 21 of these had complete $\mathrm{SCl}, 14$ an incomplete $\mathrm{SCl}-6$ of them with central cord syndrome and 1 with central cord plus Brown Sequard syndrome - and 20 patients were neurologically uncompromised.

Results: The neurophysiologic profile of the complete SCI was the absence of both m-MEPs and e-MEPs caudally to the lesion site, associated with a lack of cortical and e-SEPs cranially to the lesion site. None of these patients recovered motor function in the follow-up. A clearly detectable caudal D wave was associated with motor recovery even in deeply paraparetic patients. In one neurologically incomplete patient a reversible deterioration of m-MEPs and e-MEPs was observed during the compression-distraction manoeuvre.

Conclusion: Intraoperative neurophysiological evaluation of $\mathrm{SCl}$ patients can provide information about spinal cord function that is not retrievable by other clinical means and can correctly predict neurological outcome. Intraoperative testing during early stabilization of the spine of deeply paraparetic $\mathrm{SCl}$ patients provides additional information about their neurological profile.

Spinal Cord (2014) 52, 749-757; doi:10.1038/sc.2014.138; published online 19 August 2014

\section{INTRODUCTION}

Despite developments in neuroimaging, pre-surgical, surgical, postsurgical care and rehabilitation, spinal cord injury (SCI) remains a major cause of disability.

However, recent advances in knowledge of basic spinal cord function mechanisms have led to the development of therapeutic options that make the repair of the injured spinal cord possible. A large number of preclinical studies or clinical trials are currently in progress or developing, based on the pharmacological, biological or bioengineered approach. ${ }^{1}$ However, to test the efficacy of these new, promising treatments, a crucial point is functional evaluation of the acutely spinal cord-injured patient. Functional assessment and prognosis are at present still dominated only by clinical findings; ${ }^{2}$ nevertheless recent studies have shown that the potential for spontaneous improvement (that is, not determined by new therapies) is greater than previously reported. ${ }^{3}$

Conventional clinical neurophysiologic studies in the acute phase fail to predict recovery in a relevant percentage of patients. ${ }^{4,5}$ However, electrophysiological measures could provide information about spinal pathways that are not retrievable by clinical examination and might have additional value in prediction of functional outcome. ${ }^{6,7}$ In various animal models of experimental SCI motor evoked potentials (MEPs) and somatosensory evoked potentials
(SEPs), particularly invasive recordings, have proved to be sensitive measure of post-injury sensory motor performance. ${ }^{8-10}$ On the other hand, the last years have been testimony to the development of well-established techniques to intraoperatively assess spinal cord function during spine and spinal cord surgeries. ${ }^{11-13}$ Particularly, the combined recordings of muscle MEPs (m-MEPs) and the epidurally recorded $\mathrm{D}$ wave provide relevant prognostic information on motor outcome. ${ }^{11,14}$

Neurophysiologic techniques can be therefore applied intraoperatively with the aim to define the neurophysiologic profile of the acutely SCI patient, to identify biological marker of functionally complete spinal cord lesion and to assess chances for recovery, particularly motor outcome.

\section{MATERIALS AND METHODS}

Intraoperative recording of $\mathrm{m}$-MEPs and epidural $\mathrm{D}$ wave along with cortical (c-SEPs) and epidural (e-SEPs) SEPs was attempted in 55 patients during posterior stabilization surgery for SCI. In all, 21 patients had a complete SCI, 14 an incomplete SCI -6 of which with central cord syndrome and 1 patient with central cord plus Brown Séquard syndrome-and 20 patients were neurologically uncompromised. All patients gave informed consent after being told that potential risks included: seizures, skin burns from stimulating electrodes, tongue bites, cardiac arrhythmias, inadvertent injury caused by transcranial electrical stimulation-induced movement, epidural bleeding or

\footnotetext{
${ }^{1}$ Section of Clinical Neurophysiology, CTO, Torino, Italy; ${ }^{2}$ Department of Neurosciences, Città della Salute e della Scienza, Torino, Italy; ${ }^{3}$ Neurosurgery, CTO, Torino, Italy; ${ }^{4}$ Neurosurgery, Department of Neurological and Movement Sciences, University Hospital, Verona, Italy and ${ }^{5}$ Department of Intraoperative Neurophysiology, St Luke's Roosevelt Hospital, Mount Sinai Health System, New York, NY, USA

Correspondence: Dr P Costa, Department of Neurosciences, Section of Clinical Neurophysiology, Città della Salute e della Scienza, CTO Hospital, via Zuretti 29, Torino 10126, Italy.

E-mail: pacst@fastwebnet.it

Received 13 January 2014; revised 13 July 2014; accepted 17 July 2014; published online 19 August 2014
} 
infections. Patients with implanted heart pacemakers or brain stimulators and subjects with SCI distal to the bony level T10 were excluded from the study. Patients were preoperatively clinically assessed, by the ASIA (American Spinal Cord Injury Association) and modified McCormick scales, within $12 \mathrm{~h}$ after surgery, 7 days after surgery and at discharge. Although McCormick scale ${ }^{15}$ has been proposed for intramedullary spinal cord tumours, it provides a quick evaluation of neurological status and can be used in other types of spinal cord pathologies (Table 1).

\section{Methods}

The anaesthetic protocol during surgery included a combination of the two drugs, Remifentanil and Propofol, with total intravenous anaesthesia. No muscle relaxants were used after patient's induction and intubation.

Two flexible three-contact platinum epidural electrodes (CEDL-3PIDINX, Ad-Tech Medical Instruments Corporation, Racine, WI, USA) were inserted cranially and caudally to the injury site through a small laminectomy or flavectomy, to monitor both epidural MEPs ( $\mathrm{D}$ wave) and SEPs during surgery. The three contacts were numbered 1-3 with electrode 1 close to the edge. Commercially available intraoperative neurophysiology system (Nicolet Endeavour; Natus Medical Incorporated, San Carlos, CA, USA) was used for SEPs stimulation and recording.

\section{Table 1 Modified McCormick's clinical/functional classification} scheme

\begin{tabular}{ll} 
Grade & Definition \\
\hline I & Intact neurologically, normal ambulation, minimal dysaesthesia \\
II & Mild motor or sensory deficit, functional independence \\
III & Moderate deficit; limitation of function, independent with external aid \\
V & Severe motor or sensory deficit, limitation of function, dependent \\
& Paraplegia or quadriplegia, even if there is flickering movement
\end{tabular}

\section{Somatosensory evoked potentials}

SEPs were elicited by a 100 - or $200-\mu$ s square-wave electrical pulse presented sequentially to median nerve at the wrist and posterior tibial nerve at the ankle (rate 4.1-7.1/s, stimulus intensity adjusted individually from 20 to $40 \mathrm{~mA}$ ). The mixed (sensory and motor) nerve potential was recorded at the popliteal fossa. To optimize the montage, monopolar needle electrodes were placed at $\mathrm{Cz}^{\prime}, \mathrm{C}^{\prime}$, $\mathrm{C}^{\prime}$ and $\mathrm{Fz}$ (international 10-20 EEG system) and the best traces among $\mathrm{Cz}^{\prime}-\mathrm{Fz}, \mathrm{C} 3^{\prime}-\mathrm{Fz}, \mathrm{C} 4^{\prime}-\mathrm{Fz}, \mathrm{C} 3^{\prime}-\mathrm{C}^{\prime}$ and $\mathrm{C}^{\prime}-\mathrm{C}^{\prime}$ were used for monitoring. The acquisition bandwidth was $1-3000 \mathrm{~Hz}$; a digital filtering (typically 20-30 to $300-500 \mathrm{~Hz}$ ) was applied to improve the quality of signal. ${ }^{16}$ Analysis time was $100 \mathrm{~ms}$; averaging was stopped manually when c-SEPs were clearly reproducible. Similar stimulation and filtering parameters were used for epidural SEPs (e-SEPs): the recording montage was electrode 3 to 2 or 2 to 1 (active to reference) for the rostral electrode and vice versa for the caudal electrode in regard to the lesion site.

\section{Muscle motor evoked potentials}

m-MEPs were elicited with a 50 - or $500-\mu$ s duration of transcranially applied trains of 3-7 electrical pulses, with interpulse interval of 2-4 ms, delivered with two corkscrew-type electrodes inserted over the motor cortex regions at $\mathrm{C} 1$, C2, C3 and C4 (international 10-20 EEG system). A C1-C2 or C3-C4 dipole was used to elicit right $\mathrm{m}$-MEPs, and vice versa. Stimuli were delivered through commercially available constant-voltage and constant-current stimulators (D185; Digitimer, Welwyn Garden City, UK; pulse width $=50 \mu$ s, voltage 200-700 V or CRO STIM 2, SGM, Split, Croatia, EU; pulse width $=500 \mu \mathrm{s}$, current $80-200 \mathrm{~mA}$ ). Responses were recorded on the same instrument used for SEPs.

Muscle responses were recorded by needle electrodes placed in muscles with a belly-tendon montage from the abductor pollicis brevis (APB) in the upper extremities and both the tibialis anterior and the abductor hallucis muscles in the lower extremities. The time base was 100-200 ms and the filter band pass of 30-150/500-3000 Hz. A bite block consisting of rolled gauze was used after induction to avoid lips or tongue bites.

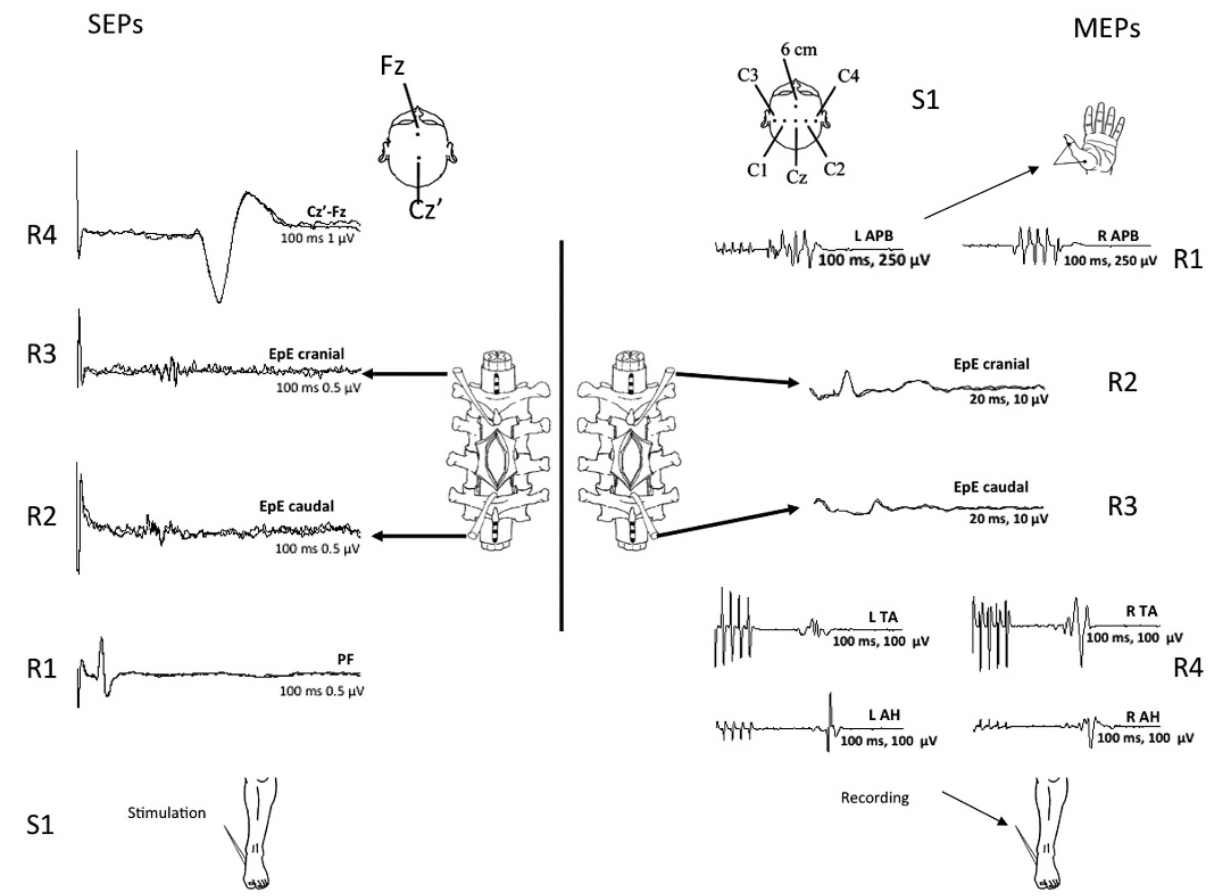

Figure 1 Neurophysiological profile of a neurologically intact patient. To the left: Spinal SEPs (R1-R4) after stimulation of the tibial nerve at the ankle (S1) recorded caudally (R2, EpE caudal) and cranially to the lesion site (R3, EpE cranial; R4, Cz'-Fz). PF=tibial nerve neurogram from the popliteal fossa. To the right: $\mathrm{D}$ wave recorded cranially (R2, EpE cranial) and caudally ( $\mathrm{R} 3, \mathrm{EpE}$ caudal) to the lesion site, and elicited by transcranial electrical stimulation (TES, S1). m-MEPs recorded from the upper (R1) and lower extremities muscles (R4). RAPB, LAPB, right and left abductor pollicis brevis muscles; RTA, LTA, right and left tibial anterior muscles; RAH, LAH, right and left abductor hallucis muscles. 
Epidural MEPs (D wave)

$\mathrm{D}$ waves were elicited by a single anodal stimulus delivered with the same stimulators as those used for m-MEPs, and recorded with the same electrodes as those for e-SEPs; filters were typically $200 / 500-3000 \mathrm{~Hz}$. Time base was $10-$ $20 \mathrm{~ms}$; in some cases an averaging of 4-10 responses was necessary to improve signal-to-noise ratio.

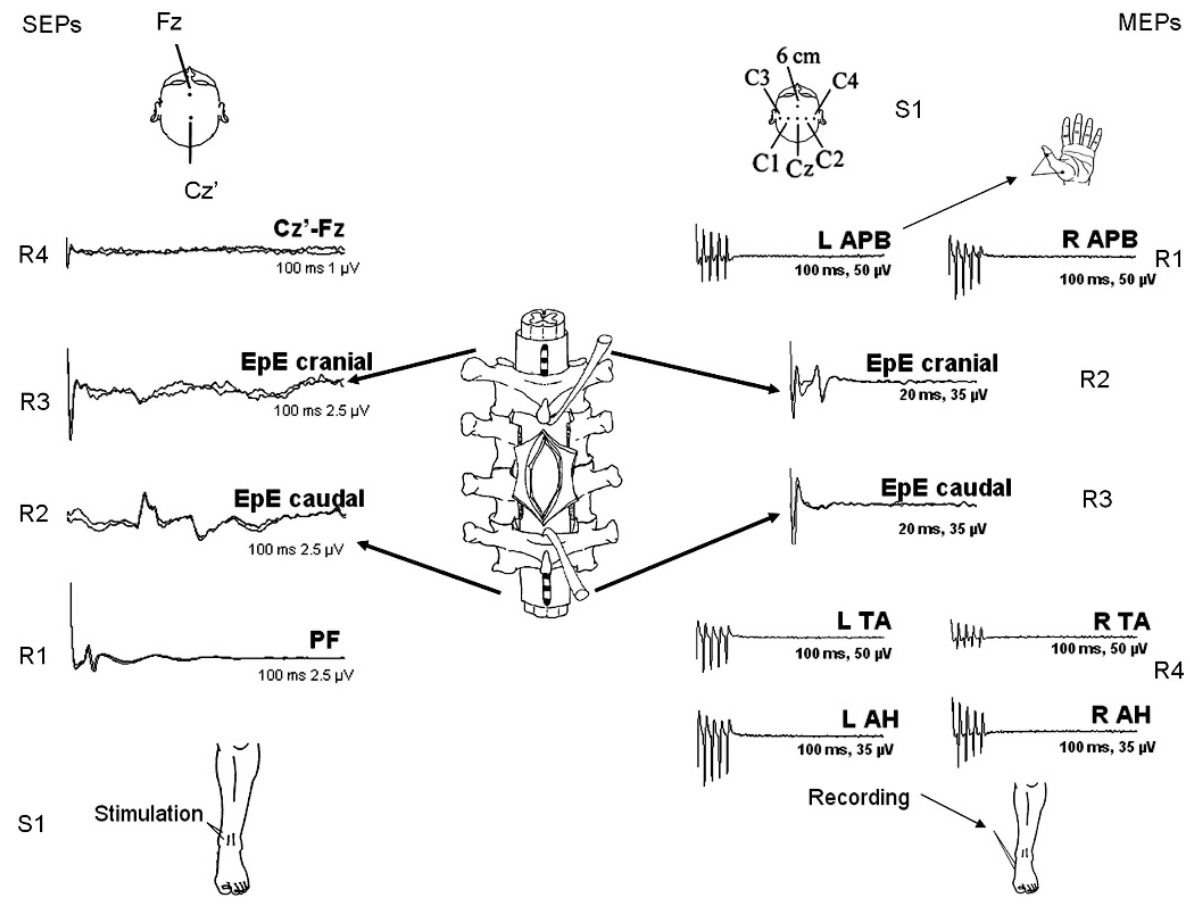

Figure 2 Typical intraoperative neurophysiologic recording in a subject with neurological complete $\mathrm{SCl}$ at cervical level. To the left: Absence of cortical (R4) and epidurally recorded spinal SEPs (R3). PF = tibial nerve neurogram from the popliteal fossa. To the right: Absence of m-MEPs from upper (R1) and lower extremities (R4), together with the presence of the $D$ wave cranially (R2) and the absence caudal to the lesion site (R3). RAPB, LAPB, right and left abductor pollicis brevis muscles; RTA, LTA, right and left tibial anterior muscles; RAH, LAH, right and left abductor hallucis muscles.

Table 2 Results in clinically complete $\mathrm{SCl}$ at time of diagnosis

\begin{tabular}{|c|c|c|c|c|c|c|c|c|}
\hline \multirow[t]{2}{*}{ No. } & \multirow[t]{2}{*}{ Sex/Age } & \multirow[t]{2}{*}{ Level } & \multirow[t]{2}{*}{ Pre-op ASIA scale } & \multicolumn{2}{|l|}{ SEPS } & \multicolumn{2}{|c|}{ MEPS } & \multirow[t]{2}{*}{ ASIA at discharge or FU } \\
\hline & & & & Cranial epidural & Scalp & Caudal D wave & Caudal m-MEPs & \\
\hline 2 & M, 20 & Thoracic & A & - & - & - & - & A \\
\hline 3 & M, 21 & Cervical & A & - & - & - & - & $A$ \\
\hline 4 & M, 74 & Thoracic & A & - & - & - & - & Deceased in ICU \\
\hline 7 & M, 69 & Thoracic & $A$ & - & - & - & - & A \\
\hline 8 & $\mathrm{~F}, 31$ & Thoracic & A & - & - & - & - & B \\
\hline 9 & $\mathrm{~F}, 78$ & Cervical & A & - & - & - & - & Deceased in ICU \\
\hline 10 & M, 74 & Thoracic & A & - & - & - & - & A \\
\hline 11 & M, 27 & Thoracic & A & - & - & - & - & B \\
\hline 16 & $\mathrm{~F}, 43$ & Thoracic & A & - & - & - & - & A \\
\hline 17 & $\mathrm{~F}, 41$ & Cervical & A & - & - & - & - & A \\
\hline 18 & $M, 48$ & Cervical & $A$ & - & - & - & - & $A$ \\
\hline 19 & M, 71 & Thoracic & A & - & - & - & - & A \\
\hline 20 & M, 55 & Cervical & A & - & - & - & - & Deceased in ICU \\
\hline 21 & M, 46 & Cervical & A & - & - & - & - & A \\
\hline
\end{tabular}

Abbreviations: Fu, follow-up; MEPs, motor evoked potentials; m-MEPs, muscle MEPs; SCI, spinal cord injury; SEPs, somatosensory evoked potentials. 
The recording montage was electrode 1 to 2 (active to reference) and/or 2 to 3 for the rostral electrode and 2 to 1 and/or 3 to 2 for the caudal electrode so as to obtain the same response polarity from both electrodes montage.

\section{RESULTS}

All uncompromised patients showed recordings of the motor and sensory evoked potentials from the upper and lower extremities within normal limits (Figure 1). No significant evoked potential modifications were observed in any case, and none of the subjects presented postoperative neurological deficits.

In all clinically complete SCI patients, the intraoperative neurophysiological profile was characterized by the absence of both $\mathrm{m}$-MEPs and $\mathrm{D}$ wave caudal to the lesion site associated with the lack of c-SEPs and e-SEPs cranially to the lesion site (Figure 2).

None of these patients recovered neurologically (Table 2). In one subject, with an ASIA-A, and C5 SCI level, the presence of m-MEPs recorded from the APB correctly predicted the recovery of hand but not leg function (Figure 3).

The intraoperative neurophysiologic pattern of neurologically incomplete SCI patients generally mirrored the clinical presentation, but a clear caudal D wave, even if with low amplitude values, was recordable in all subjects. In particular, in central cord syndrome the neurophysiological profile was characterized by the presence of the D wave caudal to the injury site but absent or low voltage m-MEPs from hand muscle (Figure 4).

Three out of fourteen neurologically incomplete patients (Table 3 ) with absent m-MEPs and a clear D wave (recorded caudal to the spinal cord lesion) recovered significantly.

One 69-year-old male (Table 3, patient no. 2), had a clinical history of liver cirrhosis and diabetes. He had minor brain trauma due to a fall (brain CT and cervical X-ray were normal). Twenty-four hours later he developed a rapid evolving tetraplegia (in a matter of hours he could move only the big toe bilaterally). A cervical MRI showed a hyperintensity at C4-C5 level. The day after, a cervical decompression
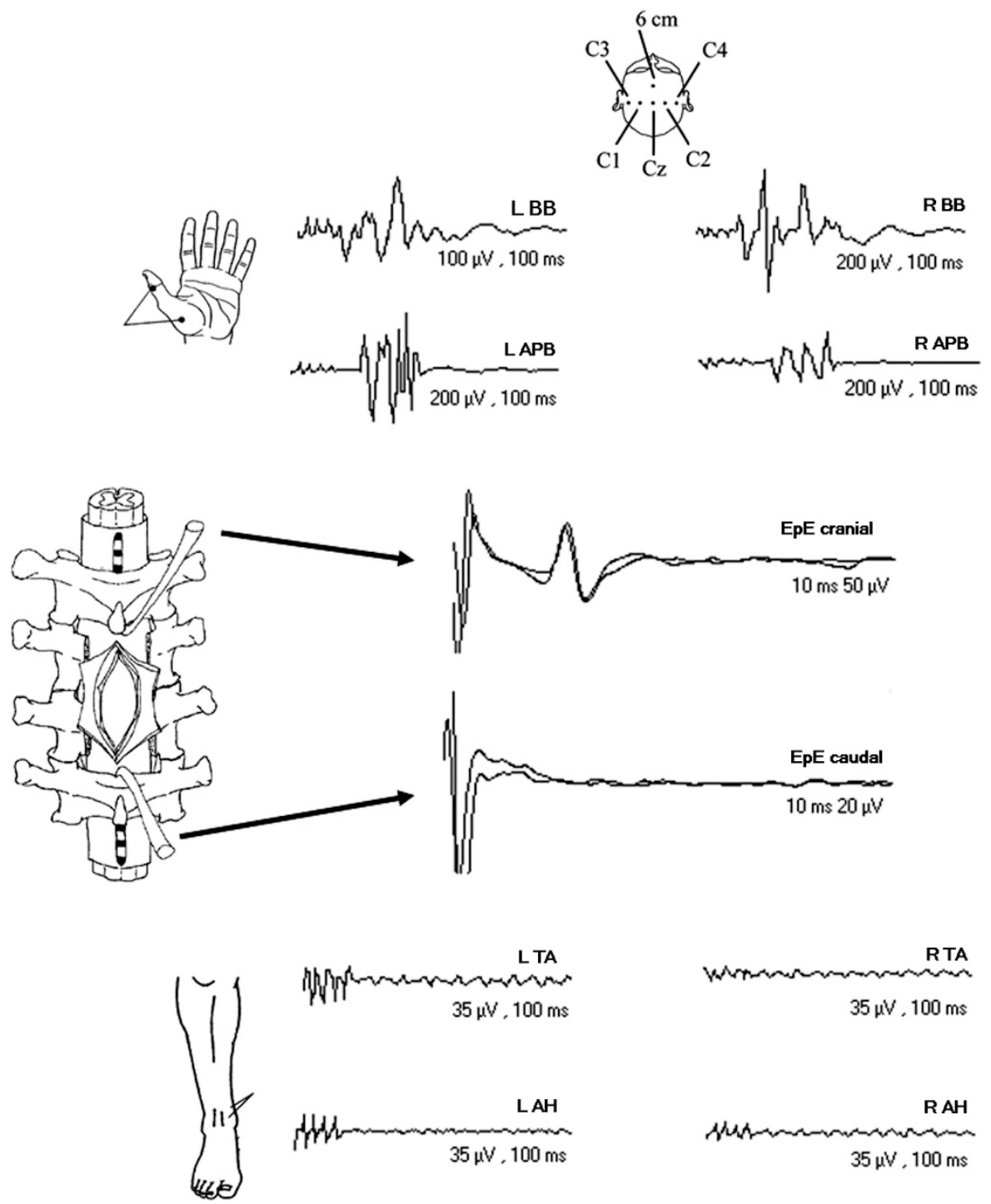

Figure 3 Intraoperative recording of MEPs in a patient with SCI at C5 and ASIA-A score at the time of surgery (Table 2, no. 14). The presence of m-MEPs from hand muscles correctly predicted recovery of hand function. RBB, LBB, right and left biceps brachii muscles; RAPB, LAPB, right and left abductor pollicis brevis muscles; RTA, LTA, right and left tibial anterior muscles; RAH, LAH, right and left abductor hallucis muscles. 


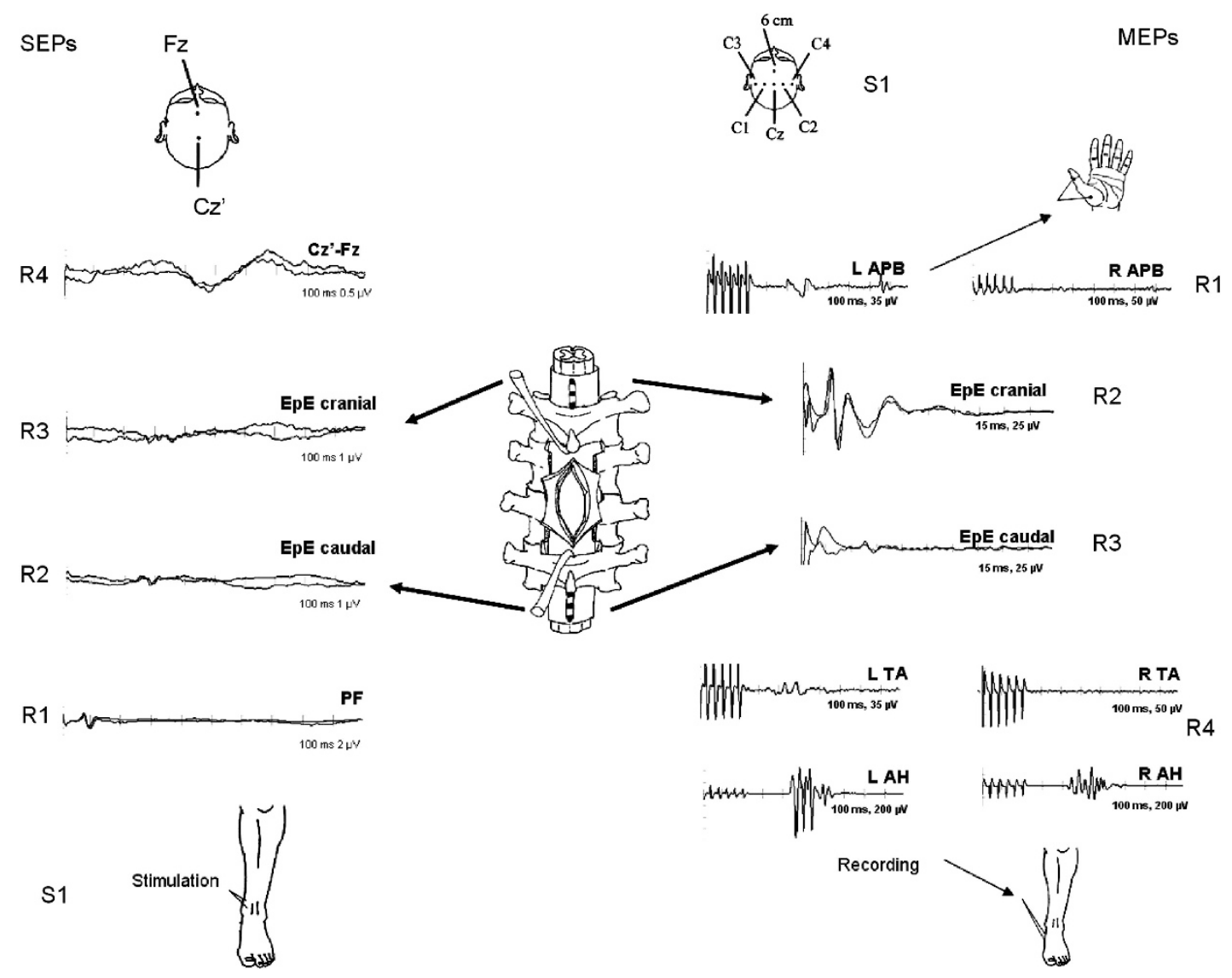

Figure 4 Patient with central cord syndrome at cervical level (Table 3, No. 5), having low amplitude m-MEPs from hand muscles (R1) with preserved m-MEPs from lower limb muscles (R4). The caudal $D$ wave is clearly recordable.

Table 3 Results in clinically incomplete $\mathrm{SCl}$ at time of diagnosis

\begin{tabular}{|c|c|c|c|c|c|c|c|c|c|c|}
\hline \multirow[t]{2}{*}{ No. } & \multirow[t]{2}{*}{ Sex/Age } & \multirow[t]{2}{*}{ Level } & \multirow[t]{2}{*}{ Pre-op ASIA scale } & \multirow{2}{*}{$\begin{array}{l}\text { Pre-op mod. } \\
\text { McKormick }\end{array}$} & \multicolumn{2}{|c|}{ SEPS } & \multicolumn{2}{|c|}{ MEPS } & \multirow{2}{*}{$\begin{array}{l}\text { Mod. McKormick } \\
\text { at discharge or FU }\end{array}$} & \multirow{2}{*}{$\begin{array}{l}\text { ASIA scale at } \\
\text { discharge or FU }\end{array}$} \\
\hline & & & & & $\begin{array}{l}\text { Cranial } \\
\text { epidural }\end{array}$ & Scalp & $\begin{array}{l}\text { Caudal } \\
D \text { wave }\end{array}$ & $\begin{array}{c}\text { Caudal } \\
\text { m-MEPs }\end{array}$ & & \\
\hline 1 & M, 70 & Cervical & D (central cord) & IV & - & - & + & + & III & $\mathrm{D}$ \\
\hline 2 & M, 69 & Cervical & C (central cord) & V & + & + & + & - & II & $\mathrm{D}$ \\
\hline 3 & $M, 44$ & Cervical & D (central cord) & II & + & + & + & + & I & $E$ \\
\hline 4 & M, 70 & Thoracic & D (central cord) & II & + & + & + & + & II & $\mathrm{D}$ \\
\hline 5 & M, 27 & Cervical & D & II & + & + & + & + & 1 & $E$ \\
\hline 6 & M, 67 & Thoracic & D & II & + & + & + & + & II & $D$ \\
\hline 7 & $\mathrm{M}, 34$ & Cervical & D (central cord) & III & + & + & + & + & II & $\mathrm{D}$ \\
\hline 8 & $F, 39$ & Cervical & D & II & + & + & + & + & 1 & $E$ \\
\hline 9 & $F, 53$ & Cervical & D & II & + & + & + & + & 1 & $E$ \\
\hline 10 & M, 36 & Cervical & C (Central cord + BS) & IV & NP & \pm & + & \pm & II & $E$ \\
\hline 11 & M, 77 & Cervical & $\mathrm{C}$ & III & + & + & + & - & II & $E$ \\
\hline 12 & $M, 74$ & Thoracic & D & II & + & + & + & - & II & $E$ \\
\hline 13 & $M, 72$ & Thoracic & C & V & + & + & + & - & II & $\mathrm{E}$ \\
\hline 14 & M, 44 & Cervical & C (central cord) & III & NP & - & + & - & II & $\mathrm{D}$ \\
\hline
\end{tabular}

Abbreviations: BS, Brown-Sequard syndrome; Fu, follow-up; MEPs, motor evoked potentials; m-MEPs, muscle MEPs; NP, not placed; SCI, spinal cord injury; SEPs, somatosensory evoked potentials; +, present; -, absent; \pm , unilaterally identifiable.

with a posterior approach was performed. Although intraoperative m-MEPs recorded from upper and lower extremities were absent, D wave recorded at the upper thoracic spinal cord was present with low amplitude and delayed latency of $10 \mathrm{~ms}$ (Figure 5). The patient quickly improved in term of days (lower extremities muscle strength was $4 / 5$, and he was able to walk with a cane in a couple of weeks). 

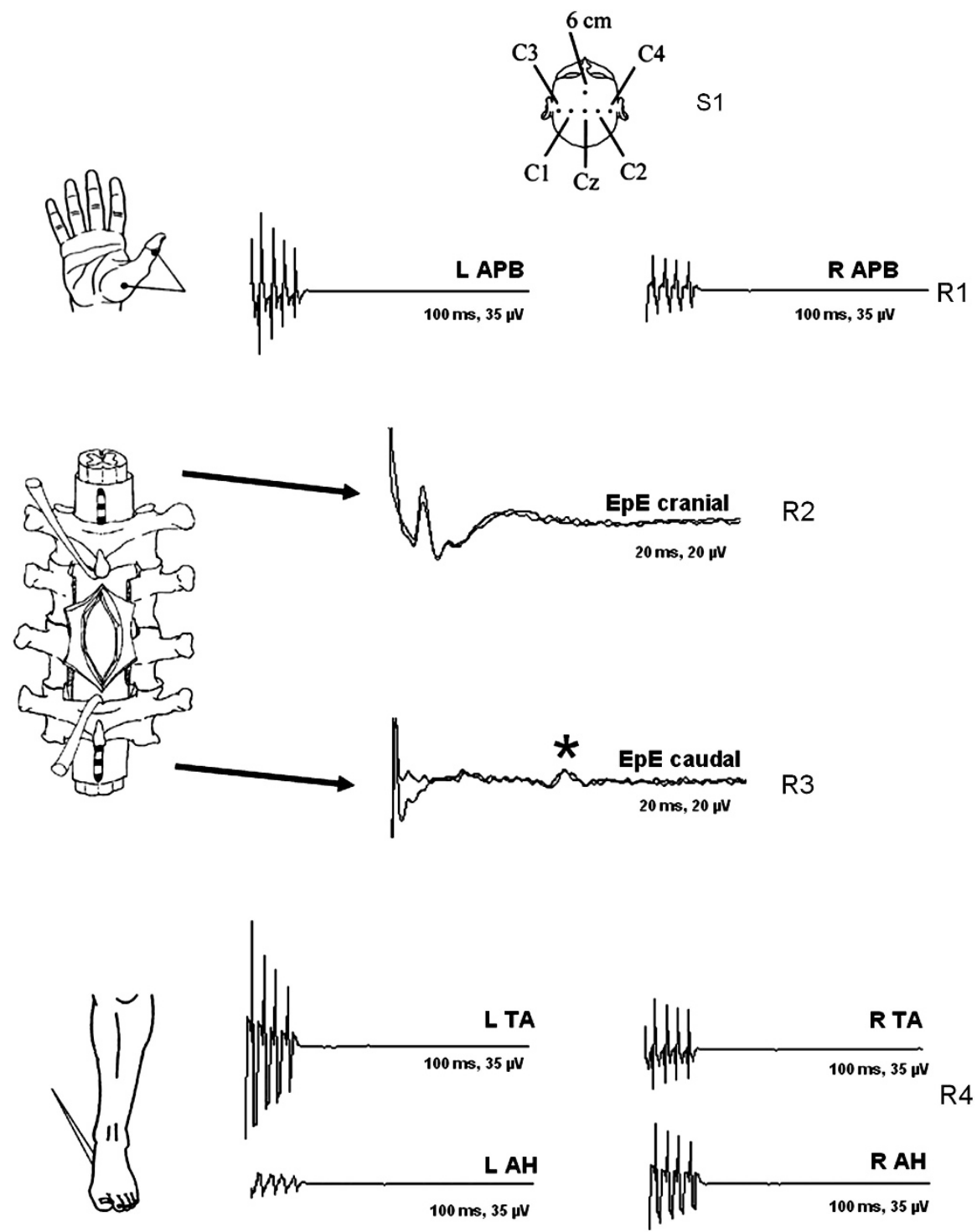

Figure 5 MEPs of patient no. 2 of Table 3, having small amplitude and delayed latency D wave $\left({ }^{*}\right)$ distally to the injured site, predicting good neurological recovery. The ptn-SEPs were bilaterally present.

Similarly another patient (Table 3, no. 13) was found deeply paraparetic (only flickering movements of the great toe were possible) due to a fall. Light touch sensation was grossly intact throughout lower extremities, whereas pinprick sensation was absent caudal to T4 level. An MRI scan showed a T2-T3 fracture dislocation with a T2-T4 spinal cord hyperintensity. Again, although m-MEPs from lower limbs were unexcitable, a clear defined caudal D wave correctly predicted a good outcome (Figure 6). The patient fully recovered in 3 months.

In 1 out of the 14 neurologically incomplete SCI patients (Table 3, patient no. 7) during surgical manoeuvre for decompression traction for spine alignment, deterioration of $\mathrm{m}$-MEPs and reduction in amplitude of the D wave occurred. m-MEPs and D wave returned to the baseline after stopping of the manoeuvre (Figure 7).

\section{DISCUSSION}

The prognosis of acute SCI, in particular motor outcome, represents a challenge for the involved health-care professionals. Nowadays the functional assessment and the magnitude of expected recovery are based on the physical examination, as tested by ASIA scoring, within $72 \mathrm{~h}$ to 1 month post trauma. ${ }^{2}$ However, as afore mentioned, recent data suggest that the rate of ASIA conversion within the first year after SCI is greater than previously reported. ${ }^{3}$ Conventional clinical neurophysiology techniques have been extensively studied in the SCI patient, but mainly in chronic phase and in association with the clinical examination. In particular both c-SEPs and m-MEPs by transcranial magnetic stimulation in the acute phase may fail to provide a correct prognosis of recovery in $\sim 25 \%$ of cases. ${ }^{4,5}$ However clinical neurophysiology methods, by their intrinsic nature, can provide (semi-) quantitative information, in terms of presence/ absence, latency and amplitude of components. ${ }^{7,17}$

Our data provide evidence in favour of an intraoperative testing of spinal cord function in SCI. In fact, intraoperative neurophysiologic reliable methods to predict functional integrity of the long tracts during surgical intervention to the spinal cord has been already developed and well established. ${ }^{11-13}$ This is particularly true for 

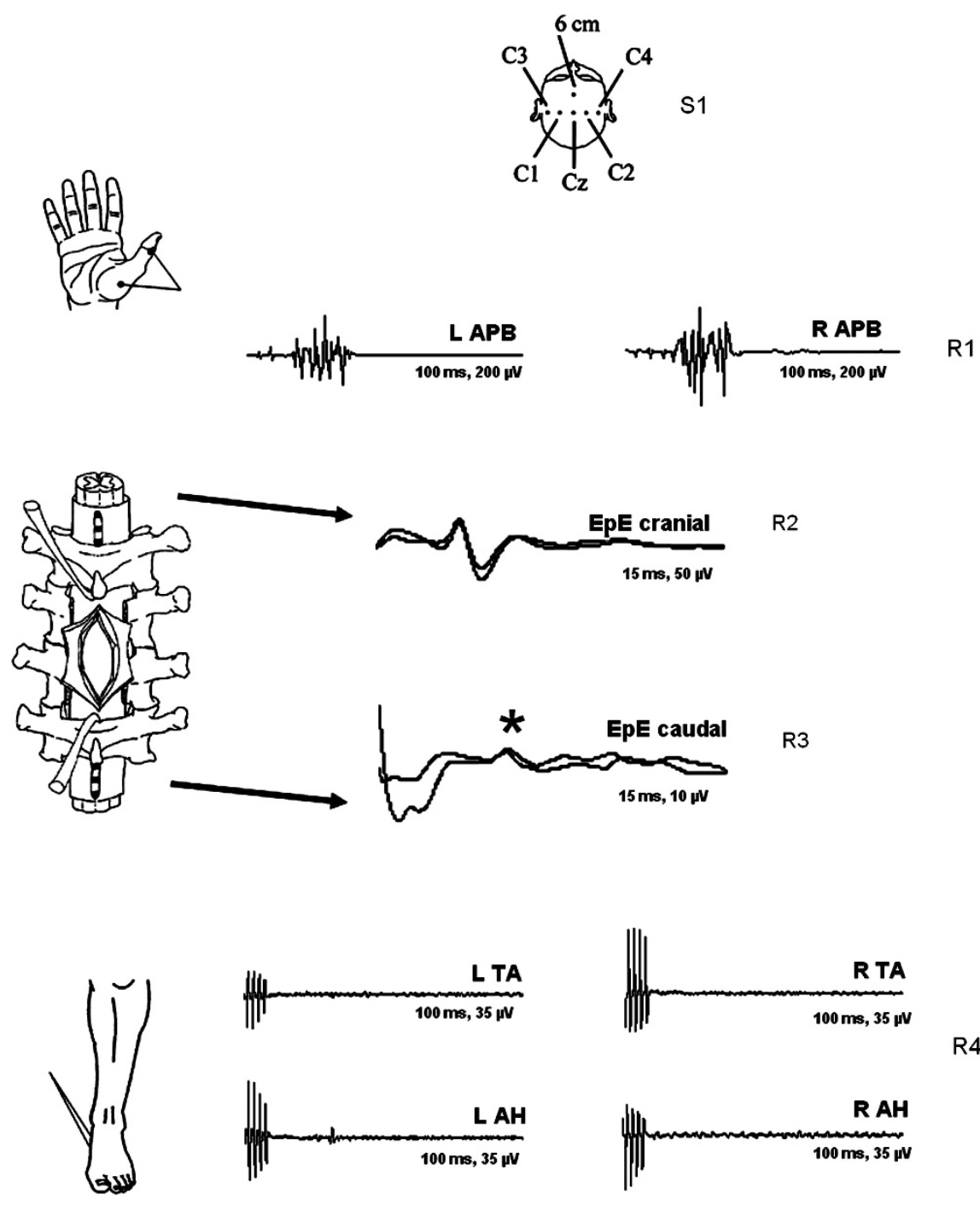

Figure 6 Intraoperative MEPs of patient 13 of Table 3. Although lower limbs muscle MEPs were absent, the recordability of caudal D wave $(*)$ correctly predicted the motor outcome. The ptn-SEPs were bilaterally present.

epidural MEPs (e-MEPs) recorded from the spinal cord in the form of $\mathrm{D}$ and I waves and MEPs recorded from the limb muscles. ${ }^{14}$ The information provided by these two techniques are complementary, being the $\mathrm{D}$ wave generated by the direct activation of axons of fast conducting fibres of the cortico-spinal tract, ${ }^{18}$ while m-MEPs depend also on the excitability of facilitatory cortical motor neurons, beyond the spinal cord conductivity and the excitability of lower motoneurons. ${ }^{14}$ In this series the absence of both m-MEPs and e-MEPs caudal to the lesion site associated with a lack of epidural and c-SEPs cranially to the lesion site has to be considered as a biological marker of a complete SCI. This is consistent with data in non-primate and primate animal models of experimental SCI. ${ }^{8-10}$

By contrast, a clearly detectable caudal $\mathrm{D}$ wave is the pre-requisite of motor recovery even in deeply paraparetic patients. In fact, the D wave is a direct measure of the number of functioning fast-conducting fibers in the corticospinal tracts. ${ }^{18}$ Since no synapses are involved between the stimulating and the recording site (the proximal axon of the cortical motoneuron is stimulated and the recording site is located caudal to the lesion site but cranially to the synapses at the $\alpha$-motoneuron), the $\mathrm{D}$ wave has been considered as the gold standard in the assessment of the integrity of the corticospinal tract. ${ }^{14}$ Many reports show that if the $\mathrm{D}$ wave is lost during the removal of an intramedullary spinal cord tumour, the patient will suffer from profound, persistent motor deficit. ${ }^{11,14,19}$ Recently Eck et al. ${ }^{20}$ reported that the possibility of recording intraoperatively evoked m-MEPs in sublesional muscles of an SCI patient correlates with a good motor outcome: this finding is similar to our patient no. 14 of Table 2. However in our series the absence of muscle MEPs below the lesion site does not correlate 'per sê with persistent loss of ambulatory capacity. In other words, the recording of m-MEPs alone may not be sufficient for a prognostic evaluation. Indeed, m-MEPs are, at times, completely lost during surgery for intramedullary spinal cord tumours but, if the D-wave amplitude is either stable or decreased by $<50 \%$, the patient will present additional transient motor deficit postoperatively but motor strength recovery after surgery, with a delay of hours or days. ${ }^{11,14,19}$ This phenomenon ('transient paraplegia') is probably due to the surgically induced temporary inactivation of non-corticospinal descending tracts and propriospinal system, while fast-conducting corticospinal fibers are mostly preserved. ${ }^{14}$ The speculative mechanism of transient 


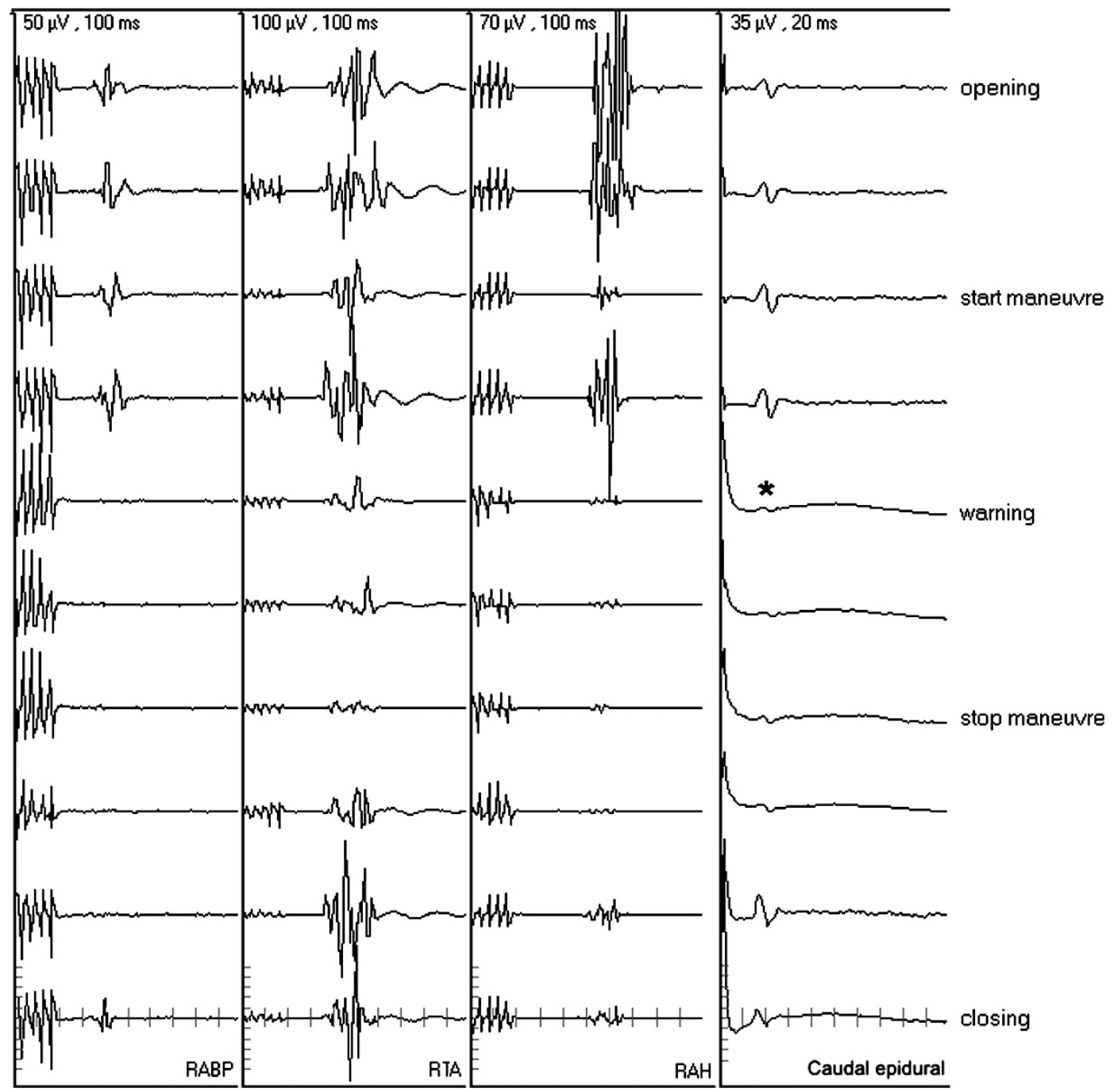

Figure 7 Neurologically incomplete spinal cord-injured patient (Table 3, patient No. 7). During surgical manoeuver for decompression traction for spine alignment deterioration of muscle MEPs and D wave $\left(^{*}\right)$ occurred. Muscle MEPs ad D wave started to return to the baseline after stopping manoeuver and completely recovered at the end of surgery. RAPB, right abductor pollicis brevis; RTA, right tibialis anterior; RAH, right abductor hallucis.

paraplegia has at least some similarity to the current hypothesis about the 'spinal shock' phenomenon. In theory, a similar phenomenon could be present in SCI and, therefore, the intraoperative recording of the $\mathrm{D}$ wave could be a potential predictive index of recovery. An experimental study reported that as few as $10 \%$ of the corticospinal fibres were sufficient to support locomotion. ${ }^{21}$ Finally, although this study is mainly focused on MEPs, SEPs retain a great value in assessing dorsal column function, such as proprioception, which is of paramount importance even for locomotion.

\section{CONCLUSIONS}

To the best of our knowledge, the combined intraoperative recording of m-MEPs and e-MEPs along with c-SEPs and e-SEPs in cases of spinal and spinal cord trauma has not been previously reported.

The intraoperative recording of $\mathrm{m}$-MEPs and e-MEPs along with c-SEPs and e-SEPs allow for a separate assessment of motor and sensory function in SCI.

In complete transaction syndrome, the intraoperative finding is characterized by the absence of both m-MEPs and e-MEPs caudal to the lesion site associated with a lack of epidural and c-SEPs cranially to the lesion site.

The presence of $\mathrm{D}$ waves beyond the lesion site generally confirms incomplete SCI for an accurate prediction of subsequent functional recovery even if $\mathrm{m}$-MEPs were absent.

In one of our cases with C5 SCI of ASIA-A, m-MEPs elicited from the completely paralyzed APB muscles correctly predicted the functional recovery of the muscles (Figure 3), which was otherwise escape prediction.

Finally, in uncompromised subjects, the intraoperative monitoring of both motor and sensory functions may reduce the risk of postoperative neurological compromise.

These data created a neurophysiologic profile of the SCI patients concerning conduction system of the spinal cord, as a background for a further expansion of neurophysiologic testing to the grey matter of the spinal cord (processing system) and further categorization, intervention and rehabilitation strategies.

\section{DATA ARCHIVING}

There were no data to deposit. 


\section{CONFLICT OF INTEREST}

The authors declare no conflict of interest.

\section{ACKNOWLEDGEMENTS}

This study would not have been possible without the technical competence and the creativity of our IOM Technologists Alessandro Borio, Marta Giacobbi, Sonia Marmolino, Maurizio Mogno, Domenico Serpella, Angela Palmitessa and Daniela Milani. The Compagnia di San Paolo Foundation, Turin, supported this study with an unrestricted grant (No. 2012.0456).

1 Wilcox JT, Cadotte D, Fehlings MG. Spinal cord clinical trials and the role for bioengineering. Neurosci Lett 2012; 519: 93-102.

2 Burns AS, Marino RJ, Flanders AE, Flett H. Clinical diagnosis and prognosis following spinal cord injury. Handb Clin Neurol 2012; 109: 47-62.

3 Spiess MR, Muller RM, Rupp R, Schuld CThe EM-SCI Study Groupvan Hedel HJA Conversion in ASIA impairment scale during the first year after traumatic spinal cord injury. J Neurotrauma 2009; 26: 2027-2036.

4 Spiess M, Schubert M, Kliesch U, Halder P. Evolution of tibial SSEP after traumatic spinal cord injury: baseline for clinical trials. Clin Neurophysiol 2008; 119 1051-1061.

5 Petersen JA, Spiess M, Curt A, Dietz V, Schubert MEM-SCI Study Group. Spinal cord injury: one-year evolution of motor-evoked potentials and recovery of leg motor function in 255 patients. Neurorehabil Neural Repair 2012; 26: 939-948.

6 Dietz V, Curt A. Neurological aspects of spinal-cord repair: promises and challenges. Lancet Neurol 2006; 5: 688-694.

7 Steeves JD, Lammertse D, Curt A, Fawcett JW, Tuszynski MH, Ditunno JF et al. Guidelines for the conduct of clinical trials for spinal cord injury (SCI) as developed by the ICCP panel: clinical trial outcome measures. Spinal Cord 2007; 45: 206-221.

8 Ross IB, Tator $\mathrm{CH}$. Further studies of nimodipine in experimental spinal cord injury in the rat. J Neurotrauma 1991; 8: 229-238.
9 Fehlings MG, Tator $\mathrm{CH}$, Linden RD, Piper IR. Motor evoked potentials recorded from normal and spinal cord-injured rats. Neurosurgery 1987; 20: 125-130.

10 Arunkumar MJ, Srinivasa Babu K, Chandy MJ. Motor and somatosensory evoked potentials in a primate model of experimental spinal cord injury. Neurol India 2001; 49: 219-224.

11 Deletis V, Sala F. Intraoperative neurophysiological monitoring of the spinal cord during spinal cord and spine surgery: a review focus on the corticospinal tracts. Clin Neurophysiol 2008; 119: 248-264.

12 MacDonald DB, Dong CCJ. Spinal cord monitoring during descending aortic procedures. In: Daube JR and Mauguiére F (eds). Handbook of Clinical Neurophysiology. Vol. 8. Intraoperative Monitoring of Neural Function [Volume Editor Nuwer MR]. Elsevier: Amesterdam, 2008, pp 815-828.

13 Nuwer MR, Packwood JW. Monitoring during the surgical treatment of scoliosis. In: Daube JR and Mauguiére F (eds). Handbook of Clinical Neurophysiology, Vol. 8. Intraoperative Monitoring of Neural Function [Volume Editor Nuwer MR]. Elsevier: Amesterdam, 2008, pp 608-617.

14 Deletis V. Intraoperative neurophysiology and methodologies used to monitor the functional integrity of the motor system. In Deletis V and Shils JL (eds). Neurophysio/ogy in Neurosurgery. Academic Press: New York, 2002, pp 25-51.

15 Shrivastava RK, Epstein FJ, Perin NI, Post KD, Jallo GI. Intramedullary spinal cord tumors in patients older than 50 years of age: management and outcome analysis. J Neurosurg Spine 2005; 2: 249-255.

16 MacDonald DB, Al-Zayed Z, Stigsby B, Al-Homoud I. Median somatosensory evoked potential intraoperative monitoring: recommendations based on signal-to-noise ratio analysis. Clin Neurophysiol 2009; 120: 315-328.

17 Curt A, Ellaway PH. Clinical neurophysiology in the prognosis and monitoring of traumatic spinal cord injury. Handb Clin Neurol 2012; 109: 63-75.

18 Patton HD, Amassian VE. Single and multiple unit analysis of cortical stage of pyramidal tract activation. J Neurophysiol 1954; 17: 345-363.

19 Sala F, Palandri G, Basso E, Lanteri P, Deletis V, Faccioli F et al. Intraoperative motor evoked potential monitoring improves outcome after surgery of intramedullary spinal cord tumor: a historical control study in 50 patients. Neurosurgery 2006; 58: 1129-1143.

20 Eck JC, Martin CJ, Lapinsky A, Connolly PJ, Dipaola C. Does intraoperative neurophysiological monitoring have predictive value for functional recovery following spinal cord injury injury? A case report. J Clin Monit Comput 2013; 27: 93-96.

21 Blight AR. Cellular morphology of chronic spinal cord injury in the cat: Analysis of myelinated axons by line sampling. Neuroscience 1983; 10: 521-543. 